\title{
APLICACIÓN DE "SERIOUS GAMES" EN EL TRATAMIENTO DE LA DEMENCIA: REVISIÓN SISTEMÁTICA
}

\author{
Trinidad Rodríguez Domínguez \\ Universidad de Extremadura, FENTO. Cáceres, España. \\ trdomin@unex.es \\ Rubén Gómez Banda \\ Universidad de Extremadura, FENTO. Cáceres, España. \\ Juan Francisco Ortega Morán \\ Centro de Cirugía de Mínima Invasión Jesús Usón, Cáceres, España. \\ Marta Santurino Muñoz \\ Universidad de Extremadura, FENTO. Cáceres, España. \\ Blas Pagador \\ Centro de Cirugía de Mínima Invasión Jesús Usón, Cáceres, España.
}

Recepción Artículo: 06 diciembre 2021

Admisión Evaluación: 06 diciembre 2021 Informe Evaluador 1: 07 diciembre 2021

Informe Evaluador 2: 07 diciembre 2021

Aprobación Publicación: 07 diciembre 2021

\section{RESUMEN}

La demencia es una enfermedad de carácter crónico y curso degenerativo que afecta de un amplio modo a la función cognitiva (capacidad de procesar el pensamiento). Debido al aumento de la esperanza de vida y a los avances técnicos en el campo de la medicina, nos encontramos ante un escenario en el que se estima que en el año 2030 el número de personas con demencia alcance los 82 millones, llegando a 152 millones en 2050 (1). Para su tratamiento se han utilizado terapias farmacológicas de éxito cuestionable. En los últimos años, los "serious games" se han abierto paso como una alternativa en el tratamiento de la demencia. Existe una abundante y diversa investigación en relación a este tema. Sin embargo, se hace necesaria una mayor homogeneidad en cuanto al diseño de las intervenciones se refiere, así como de las variables utilizadas, que facilite el futuro de la investigación en dicho campo. Por otro lado, queda patente la necesidad de la presencia de profesionales como Ios Terapeutas Ocupacionales en dichas investigaciones, los cuales pueden aportar una visión más amplia del impacto de la enfermedad en las distintas áreas ocupacionales, haciendo especial hincapié en el área del juego (2), el cual está recogido en su marco de trabajo para la práctica profesional. En esta revisión sistemática, analizamos artículos que comprenden el uso de "serious games" en el tratamiento de la demencia extrayendo los pros y contras, y sugiriendo pequeños cambios que pueden mejorar la investigación en dicho campo.

Palabras clave: demencia; Serious games; terapia ocupacional 


\title{
APLICACIÓN DE "SERIOUS GAMES” EN EL TRATAMIENTO DE LA DEMENCIA: REVISIÓN SISTEMÁTICA
}

\begin{abstract}
Application of serious games in the treatment of dementia: a systematic review. Dementia is a chronic disease with a degenerative course that broadly affects cognitive function (ability to process thought). Due to the increase in life expectancy and technical advances in the field of medicine, we are facing a scenario in which it is estimated that in 2030 the number of people with dementia will reach 82 million, reaching 152 million in 2050 (1). Pharmacological therapies have been used for their treatment with questionable success. In recent years, "serious games" have made their way as an alternative in the treatment of dementia. There is abundant and diverse research on this topic. However, there is a need for greater homogeneity in the design of interventions, as well as the variables used, to facilitate future research in this field. On the other hand, there is a clear need for the presence of professionals such as occupational therapists in such research, who can provide a broader view of the impact of the disease in the different occupational areas, with special emphasis on the area of play (2), which is included in their framework for professional practice. In this systematic review, we analyse articles involving the use of serious games in the treatment of dementia, extracting the pros and cons, and suggesting small changes that may improve research in this field.
\end{abstract}

Keywords: dementia; Serious games; occupational therapy

\section{INTRODUCCIÓN}

La demencia es un síndrome de naturaleza crónica o progresiva en la amplia mayoría de los casos, que se caracteriza por un cuadro de deterioro en lo que comprende a la función cognitiva (capacidad de procesar el pensamiento) con mayor impacto de lo que consideramos consecuencias del envejecimiento natural (1). Afecta de diverso modo al pensamiento, la memoria, la comprensión, la orientación, la capacidad de aprendizaje, el juicio, el cálculo y el lenguaje. Dicho deterioro de las funciones cognitivas suele ir acompañado (y precedido en ocasiones) por el deterioro de la motivación, el control emocional y el comportamiento social condicionando la gravedad del cuadro clínico (3).

Existen amplias y diversas clasificaciones en torno a la demencia. Los criterios de clasificación más empleados en la actualidad hacen referencia a las características clínicas del síndrome demencial y su etiología (3). De las más relevantes y utilizadas dentro de la práctica clínica encontramos las diseñadas atendiendo a la fase evolutiva del síndrome demencial. La mayor parte de ellas derivan del estudio evolutivo de la enfermedad de Alzheimer, siendo incierta su aplicación a otras patologías. Las más importantes son la "Clinical Dementia Rating Scale" (CDR) (4) y la "Global Deterioration Scale" (GDS) (5).

La escala GDS realiza una descripción en siete fases (GDS1-GDS7), que comprende desde la ausencia de alteraciones a las fases más graves de la enfermedad de Alzheimer (6). En el estadio 1 (GDS1) no existe el deterioro cognitivo. En el 2 (GDS2) presentan manifestaciones subjetivas de pérdida de memoria por parte del individuo. El estadio 3 (GDS3) refiere a deterioro cognitivo leve, pequeños olvidos de nombre y ubicación de objetos cotidianos. El estadio 4 (GDS4) corresponde al deterioro cognitivo moderado (demencia leve), en la que se aprecian dificultades evidentes en la evocación y problemas en la memoria de trabajo y a corto plazo. Los estadios 5 y 6 (GDS5-GDS6) corresponden con las fases moderada y moderadamente grave de la enfermedad, donde se presentan dificultades manifiestas para recordar aspectos importantes, existiendo también desorientación espaciotemporal. El estadio 7 (GDS7) corresponde con la fase grave, en la que el usuario depende totalmente de su cuidador, pudiendo presentarse conductas delirantes y pérdida de la capacidad verbal.

Además, la escala GDS se complementa con la escala "Functional Assessment Staging" (FAST) para evaluar de un modo más exhaustivo la demencia severa en las fases GDS6 y GDS7 (7). La fase GDS6 se subdivide en cinco sub-fases que marcan una disminución en la habilidad para: a) el vestido, b) el baño, c) el inodoro, d) incontinencia urinaria, y e) incontinencia fecal. La fase GDS7 se subdivide en 6 sub-fases correspondientes a: a) reducción del habla a 1-5 palabras por día, b) pérdida del vocabulario, c) pérdida de la marcha, d) incapacidad de mantener la sedestación, e) incapacidad de sonreír, y f) incapacidad de mantener la cabeza erguida. 
Debido al aumento de la esperanza de vida derivada de los avances técnicos en el campo de la medicina y a la disminución de las tasas de natalidad derivadas de los nuevos modelos socioeconómicos en todo el mundo, nos encontramos ante un escenario en el que la "población envejecida" y sus problemas de salud son fuente continua de investigación. Entre ellos encontramos la demencia y su tratamiento, según la Organización Mundial de la Salud (OMS) la demencia afecta a unos 50 millones de personas, de las cuales alrededor del 60\% viven en países de ingresos medios y bajos, y se estima que cada año aparecen alrededor de 10 millones de nuevos casos (1). Se calcula que entre un $5 \%$ y un $8 \%$ de la población general de 60 años o más sufre demencia en un determinado momento. Además, las previsiones estiman que el número total de personas con demencia alcance los 82 millones en 2030 y llegue hasta los 152 millones en 2050. En los comienzos, la investigación sobre el tratamiento de la demencia se centró única y exclusivamente en tratamientos farmacológicos, los cuales no previenen la enfermedad de manera eficaz, ni ofrecen un tratamiento etiopatogénico que cure o detenga la enfermedad (8). Actualmente disponemos de tratamientos sintomáticos modestamente efectivos, para mejorar la cognición y las alteraciones de conducta con el fin de mantener la función. A raíz de esos resultados, en los últimos años las investigaciones han adoptado una tendencia que centra su foco de atención en terapias no farmacológicas como los "serious games".

Los "serious games" son juegos que no tienen como objetivo principal el entretenimiento, disfrute o diversión (9), sino que sus objetivos se centran mayoritariamente en la mejora o mantenimiento de la salud del usuario (10). Además, existe evidencia de que la terapia con "serious games" obtiene mejores resultados en las capacidades cognitivas de los usuarios que otras terapias como el tratamiento farmacológico y la estimulación cognitiva tradicional (11).

El término "serious games", al contrario de lo que se podría pensar, apareció mucho antes de la proliferación de las tecnologías informáticas (12). En los años 70 el autor Clark Abt la utilizó para su libro de título homónimo "serious games", en el que formuló una de las primeras definiciones: "reducido a su forma esencial, un juego es una actividad entre dos o más jugadores independientes que tratan de alcanzar sus objetivos en un contexto limitado. Una definición más convencional diría que un juego es un contexto con reglas entre contrincantes que tratan de ganar objetivos, refiriéndonos a ellos en el sentido de que estos juegos tienen una finalidad educativa explícita y cuidadosamente pensada, y no están pensados para ser jugados principalmente por diversión".

Los precursores de los "serious games" eran de carácter no digital y su uso estaba restringido a unas determinadas áreas como son: entrenamiento militar, el ámbito educativo y la política (13). A medida que las tecnologías informáticas se fueron abriendo paso en la materia aparecieron definiciones más ajustadas a lo que hoy en día conocemos por "serious games", como la que propuso Michael Zyda (13), quien participó en el desarrollo de "America s army", un serious game utilizado en el entrenamiento del ejército de los Estados Unidos: "Un desafío mental, jugado con un ordenador de acuerdo con reglas específicas, que utiliza el entretenimiento, para promover objetivos de formación, educación, salud, política pública y comunicación estratégica del gobierno o de la empresa."

En lo que concierne al campo de cuidado de la salud, uno de los primeros "serious games" que apareció fue "Captain Novolin" (1992) (14), diseñado para enseñar a los niños a controlar la diabetes. Este juego de plataformas hace uso de "bonificaciones" para transmitir su mensaje de cuidado de los niveles de glucosa. Dichas bonificaciones son artículos de comida que el protagonista puede recoger, y si el protagonista excede el número de alimentos recogidos o su valor nutricional, corre el riesgo de enfermar debido a un alto nivel de glucosa en sangre. Dado el éxito de dicho juego, la misma empresa (Raya Systems) desarrolló otro juego llamado "Packy \& Marlon" (15) similar a "Captain Novolin" que fue utilizado en un ensayo clínico por Brown et al. en 1997 obteniendo unos resultados sorprendentes en los usuarios a los que se les presentó el juego: los usuarios controlaron mejor su diabetes y los desplazamientos al hospital derivados de una crisis de glucosa disminuyeron en un $77 \%$.

En lo relativo a los tratamientos no farmacológicos para el tratamiento de la demencia, la Terapia Ocupacional, como disciplina del área socio-sanitaria, busca la promoción del bienestar y la mejora de los indicadores de salud a través de la ocupación (16). Uno de los principales roles de esta disciplina a la hora de inter- 


\section{APLICACIÓN DE "SERIOUS GAMES" EN EL TRATAMIENTO DE LA DEMENCIA: REVISIÓN SISTEMÁTICA}

venir con usuarios con demencia corresponde al desempeño ocupacional, potenciando y promoviendo el uso de aquellas habilidades que aún permanecen intactas (17). La intervención se desarrolla en contextos diversos, entre Ios que cabe destacar: atención domiciliaria, estimulación psicomotora, programas de apoyo, modificación del entorno, atención primaria y programas de apoyo y estimulación de la función cognitiva. En estos últimos, que hacen referencia a la estimulación de la función cognitiva, uno de los principales problemas que nos encontramos es el descenso de la motivación de los usuarios debido a que la mayoría de actividades y tareas propuestas consisten en la repetición de acciones que terminan por aburrir al usuario, y como consecuencia la terapia pierde potencial y efectividad (18). Para tratar de evitar que situaciones como esa influyan en el proceso terapéutico, el Terapeuta Ocupacional debe contar con un amplio repertorio de actividades y materiales que en muchas ocasiones le son difíciles de conseguir. Por ello, los "serious games" se presentan no solo como una herramienta a la que acudir en un momento determinado, si no como un aliado con el que contar en cualquier intervención, debido a que se ha demostrado que poseen múltiples beneficios como: mejora de la capacidad de autogestión de la enfermedad, mayor adhesión al tratamiento, aumento de satisfacción y autoestima, mejora de la motivación extrínseca e intrínseca y refuerza la implicación del usuario por modificar su conducta (19).

Este estudio pretende por tanto realizar una revisión bibliográfica con el objetivo de: a) identificar, analizar y resumir la investigación que implique el uso de "serious games" en el tratamiento de personas con demencia, y b) estudiar la figura del Terapeuta Ocupacional en la intervención con "serious games" aplicados a la demencia.

\section{METODOLOGíA}

La metodología empleada para la realización de la presente revisión bibliográfica se especifica a continuación en términos de: Estrategia de búsqueda y Criterios de inclusión y exclusión.

\section{Estrategia de búsqueda}

Las búsquedas sistemáticas se realizaron en las siguientes bases de datos: Pubmed, Scopus y Web of Science. Las búsquedas se limitaron a los artículos revisados por pares en inglés. En las tres bases de datos se combinaron los términos "dementia" y "serious games" utilizando el operador booleano "AND". Los títulos y resúmenes de los estudios resultantes fueron revisados para comprobar los artículos que podían ser incluidos en base a los criterios de inclusión y exclusión. La búsqueda se centró en estudios realizados entre 2015 y marzo de 2021.

\section{Criterios de inclusión y exclusión}

Como criterios de inclusión, se han tenido en cuenta artículos cuya fecha de publicación sea a partir de 2015 (inclusive), de texto gratis completo, y en los que se realice alguna intervención con sujetos. Como criterio de exclusión, no se han tenido en cuenta las revisiones bibliográficas.

\section{Diagrama Prisma}

En la figura 1 se muestra el proceso de selección de artículos incluidos para ser analizados en la revisión bibliográfica según el diagrama Prisma. 
Figura 1. Diagrama Prisma
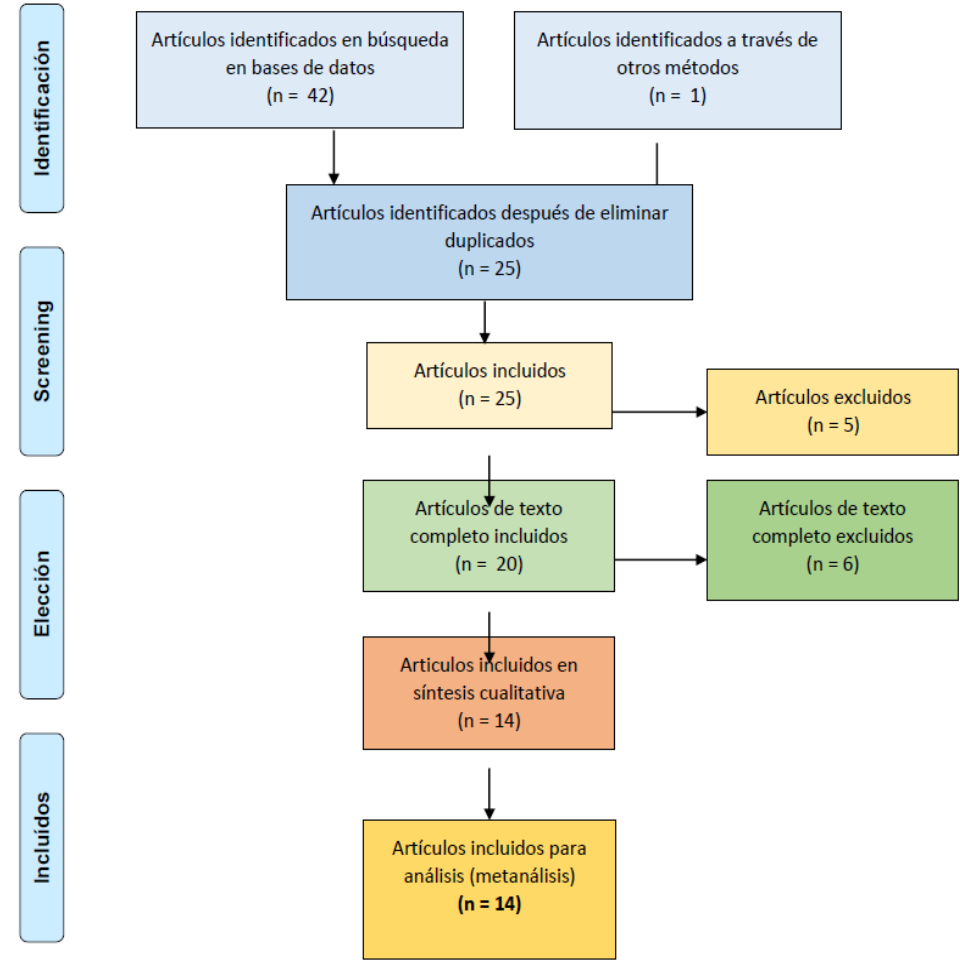


\section{RESULTADOS}

En la tabla 1 se muestra un análisis descriptivo de los artículos incluidos.

Tabla 1. Análisis descriptivo de artículos

\begin{tabular}{|c|c|c|c|c|c|}
\hline Autor & Año & País & Enfermedad & $\begin{array}{l}\text { Tipo de } \\
\text { estudio }\end{array}$ & $\begin{array}{l}\text { Edad } \\
\text { (años) }\end{array}$ \\
\hline $\begin{array}{l}\text { 1. Tziraki et } \\
\text { al. (20) }\end{array}$ & 2017 & $\begin{array}{l}\text { Canadá } \\
\text { e Israel }\end{array}$ & Demencia & $\begin{array}{c}\text { Estudio } \\
\text { piloto }\end{array}$ & $>65$ \\
\hline $\begin{array}{l}\text { 2. Fasilis et } \\
\text { al. (21) }\end{array}$ & 2018 & Grecia & Demencia & $\begin{array}{c}\text { Estudio de } \\
\text { casos }\end{array}$ & $\begin{array}{c}73.6 \\
\text { (media) }\end{array}$ \\
\hline $\begin{array}{l}\text { 3. Robert et } \\
\text { al. }(22)\end{array}$ & 2020 & Francia & $\begin{array}{l}\text { Alzheimer y } \\
\text { trastornos } \\
\text { neurocognitiv } \\
\text { os mixtos }\end{array}$ & $\begin{array}{c}\text { Ensayo } \\
\text { Clínico } \\
\text { Aleatorizad } \\
\text { o }\end{array}$ & $\begin{array}{c}79.4 \\
\text { (media) }\end{array}$ \\
\hline $\begin{array}{l}4 . \\
\text { Amaefule et } \\
\text { al. (23) }\end{array}$ & 2020 & $\begin{array}{c}\text { Aleman } \\
\text { ia }\end{array}$ & Demencia & $\begin{array}{c}\text { Ensayo } \\
\text { clínico } \\
\text { aleatorizado } \\
\text { simple } \\
\text { ciego } \\
\text { (piloto) }\end{array}$ & $60-85$ \\
\hline $\begin{array}{l}\text { 5. Yun et al. } \\
\text { (24) }\end{array}$ & 2020 & $\begin{array}{l}\text { Corea } \\
\text { del Sur }\end{array}$ & $\begin{array}{c}\text { Demencia y } \\
\text { deterioro } \\
\text { cognitivo } \\
\text { leve } \\
\end{array}$ & $\begin{array}{l}\text { Estudio } \\
\text { piloto de } \\
\text { viabilidad }\end{array}$ & $\geq 65$ \\
\hline $\begin{array}{l}\text { 6. Burdea et } \\
\text { al. }(25)\end{array}$ & 2015 & $\begin{array}{c}\text { Estados } \\
\text { unidos }\end{array}$ & $\begin{array}{c}\text { Demencia y } \\
\text { ACV }\end{array}$ & $\begin{array}{l}\text { Estudio de } \\
\text { viabilidad }\end{array}$ & $55-73$ \\
\hline $\begin{array}{l}\text { 7. Vallejo et } \\
\text { al. (26) }\end{array}$ & 2017 & Suiza & Alzheimer & $\begin{array}{c}\text { Estudio de } \\
\text { usabilidad y } \\
\text { potencial de } \\
\text { cribado } \\
\end{array}$ & 74-78 \\
\hline $\begin{array}{l}\text { 8. Swinnen } \\
\text { et al. (27) }\end{array}$ & 2021 & $\begin{array}{l}\text { Bélgica } \\
\text {, Suiza, } \\
\text { Suecia, } \\
\text { Reino } \\
\text { Unido, } \\
\text { Australi } \\
\text { a }\end{array}$ & $\begin{array}{c}\text { Trastorno } \\
\text { neurocognitiv } \\
\text { o mayor }\end{array}$ & $\begin{array}{c}\text { Ensayo } \\
\text { Clínico } \\
\text { Aleatorizad } \\
\text { o (piloto) }\end{array}$ & $\geq 65$ \\
\hline $\begin{array}{l}9 . \\
\text { Hassandra } \\
\text { et al. (28) }\end{array}$ & 2021 & Grecia & $\begin{array}{c}\text { DCL } \\
\text { (Deterioro } \\
\text { cognitivo } \\
\text { leve) }\end{array}$ & $\begin{array}{l}\text { Estudio de } \\
\text { viabilidad } \\
\text { de método } \\
\text { mixto } \\
\end{array}$ & $\begin{array}{c}73.22 \\
\text { (media) }\end{array}$ \\
\hline $\begin{array}{l}\text { 10. Chen et } \\
\text { al. (29) }\end{array}$ & 2021 & Taiwán & $\begin{array}{l}\text { DCL y } \\
\text { demencia } \\
\text { moderada- } \\
\text { grave }\end{array}$ & $\begin{array}{l}\text { Estudio pre- } \\
\text { post }\end{array}$ & $\begin{array}{c}75.38 \\
\text { (media) }\end{array}$ \\
\hline $\begin{array}{l}\text { 11. Iliadou } \\
\text { et al. (30) }\end{array}$ & 2021 & Grecia & $\begin{array}{c}\text { DCL } \\
\text { amnésico y } \\
\text { adultos sanos }\end{array}$ & $\begin{array}{c}\text { Estudio } \\
\text { comparativo }\end{array}$ & $\begin{array}{c}66.09 \\
\text { (media) }\end{array}$ \\
\hline
\end{tabular}




\begin{tabular}{|l|c|c|c|c|c|}
\hline $\begin{array}{l}\text { 12. Skikos } \\
\text { et al. (31) }\end{array}$ & 2021 & Grecia & DCL & $\begin{array}{c}\text { Estudio de } \\
\text { viabilidad }\end{array}$ & $\begin{array}{c}72 \\
(\mathrm{media})\end{array}$ \\
\hline $\begin{array}{l}\text { 13. Ge et al. } \\
\text { (32) }\end{array}$ & 2021 & China & $\begin{array}{c}\text { DCL y } \\
\text { Alzheimer }\end{array}$ & $\begin{array}{c}\text { Estudio pre- } \\
\text { post }\end{array}$ & $\begin{array}{c}25.33 \\
(\mathrm{~m}) \\
63(\mathrm{~m})\end{array}$ \\
\hline $\begin{array}{l}\text { 14. Bojan et } \\
\text { al. (33) }\end{array}$ & 2021 & $\begin{array}{c}\text { Reino } \\
\text { Unido } \\
\mathrm{y} \\
\text { Grecia }\end{array}$ & $\begin{array}{c}\text { Demencia y } \\
\text { Alzheimer }\end{array}$ & $\begin{array}{c}\text { Estudio } \\
\text { piloto }\end{array}$ & $\begin{array}{c}74.56 \\
73.63 \\
\text { (media) }\end{array}$ \\
\hline
\end{tabular}

A continuación se detalla el análisis explicativo de los artículos:

Tziraki et al. (20) realizaron una intervención de 102 sesiones por semana de 20-30 minutos a 38 personas (24 con demencia, 14 sanos) durante 10 semanas (frecuencia: 2 veces por semana). Aplicaron las escalas "Montreal Cognitive Assessment" (MoCA) y "Mini-Mental State Examination" (MMSE), y analizaron la variable de velocidad de procesamiento. Los resultados muestran una mejora de la velocidad de finalización de las pantallas para ambos grupos $(p=0.02)$.

Fasilis et al. (21) realizaron una intervención con 3 sesiones de familiarización (1h), entrenamiento y fase final sobre una muestra de 10 personas durante 7 semanas. Aplicaron las escalas "Digit Span Forward \& Backward", prueba de recuerdo de historias de Babcock, Torre de Hanoi, prueba de clasificación de tarjetas de Wisconsin x64 y FAB, y analizaron las variables de memoria, memoria de trabajo, atención, resolución de problemas, pensamiento rígido y funciones ejecutivas. Los resultados muestran una mejora de la memoria $(p=0.009)$ y de las funciones ejecutivas ( $p=0.010$ ), pero no existe mejora estadísticamente significativa en las demás áreas.

Robert et al. (22) realizaron una intervención con un grupo MeMo (4 sesiones (30 min) por semana) y un grupo control (4 sesiones (30 min) por semana) sobre 46 personas durante 12 semanas (frecuencia: sesiones de 30 min, 4 veces por semana). Aplicaron las escalas MMSE, Cuestionario del Informante sobre el Declive Cognitivo en los Ancianos (IQCODE), prueba de realización de ensayos A (TMT A), prueba de Stroop, prueba de sustitución de símbolos digitales (DSST), batería de evaluación frontal (FAB), Inventario Neuropsiquiátrico (NPI) e Inventario de Apatía (Al; versión clínica), y analizaron las variables de rendimiento cognitivo y motivación. Los resultados indican que no existe mejora estadísticamente significativa en el rendimiento cognitivo y hay un aumento de la apatía $(p=0.01)$ en el grupo NO activo

Amaefule et al. (23) realizaron una intervención mediante una tarea de navegación del mundo real a un sistema virtual basado en una cinta rodante para el análisis de la marcha con 13 personas durante 2.5 horas. Aplicaron las escalas MMSE y Cuestionario de Presencia del Grupo (IPQ), y analizaron las variables grado de inmersión, orientación, frecuencia cardiaca y conductividad cutánea. Los resultados muestran un grado considerable de inmersión, 37.5 media de casos de desorientación en el grupo demencia, 1 de 4 pacientes (25\%) con demencia mostró fatiga temprana en la navegación, y no existen cambios estadísticamente significativos en cuanto a frecuencia cardiaca y conductividad cutánea.

Yun et al. (24) realizaron una intervención con 4 juegos de cosecha y 3 de cocina con 11 pacientes con demencia y deterioro cognitivo leve en una sesión única de 30 minutos. Aplicaron las escalas MMSE y CDR, y analizaron las variables viabilidad, usabilidad, tiempo de reacción y velocidad motora. Los resultados indican satisfacción general (5.64 en escala Likert 1-7), el tiempo de reacción disminuyó sin cambios estadísticamente significativos y no existen cambios estadísticamente significativos en velocidad motora.

Burdea et al. (25) realizaron una intervención con Terapia BrightBrainer (20 minutos de juego real por sesión en las semanas 1 y 2, 25 minutos en las semanas 3 y 4, a 30 minutos (semanas 4 y 6 ) y a 40 minutos en las dos últimas semanas de rehabilitación) con 10 personas durante 8 semanas (frecuencia: 2 sesiones por semana). Aplicaron las escalas Inventario de depresión de Beck, Batería de Evaluación neuropsicológica, Módulo de Atención (Orientación, Espacio de Dígitos y Puntos) y el Módulo de Funcionamiento Ejecutivo (subprueba de 
Generación), Test de Aprendizaje Verbal de Hopkins, Test de Memoria Visoespacial Breve y Test de Creación de Rutas, y analizaron las variables toma de decisiones, depresión, velocidad de procesamiento y atención auditiva. Los resultados muestran una mejora en toma de decisiones $(p<0.01)$ y en la depresión $(p<0.056)$, y mejora no estadísticamente significativa en la velocidad de procesamiento $(p<0.17)$ y en atención auditiva $(p<0.17)$.

Vallejo et al. (26) realizaron una intervención de simulación de situaciones de la vida cotidiana con seis tareas (tres tareas de navegación, una tarea de compra, una tarea de cocina y una tarea de preparación de la mesa) con 38 personas en 1 sesión (de tiempo indeterminado). Aplicaron las escalas MoCA, Escala de Actividades de la Vida Diaria de Bristol, Cuestionario de Actividades Funcionales y Escala de Depresión Geriátrica, y analizaron las variables de tiempo en completar la tarea y prueba t de muestras independientes (usabilidad). Los resultados indican que los usuarios del grupo con Alzheimer tardaron más en completar las tareas que el grupo de controles sanos. En cuanto a la medida objetiva de usabilidad no existen diferencias significativas entre los pacientes y los controles sanos.

Swinnen et al. (27) realizaron una intervención con un grupo tratamiento (sesiones de 15 min en la plataforma Dividat Senso) y un grupo control (visionado y escucha de música y vídeos de su preferencia 3 veces por semana, durante ocho semanas en sesiones de $15 \mathrm{~min}$ ) con 45 personas. Aplicaron las escalas Batería Breve de Rendimiento Físico (SPPB), MoCA, NPI, Escala de Cornell para la Depresión en la Demencia (CSDD) y Cuestionario de Calidad de Vida en la Demencia (DQoL), y analizaron las variables atención dividida, atención selectiva, flexibilidad, control postural, memoria de trabajo visoespacial, marcha y síntomas de depresión. Los resultados muestran una mejora en el funcionamiento general de las extremidades inferiores $(p<0.001)$, en la atención y la memoria de trabajo visoespacial medida con la escala MoCA $(p=<0.001)$ y en los síntomas de depresión medidos con la escala CSDD $(p<0.001)$, pero no se encontraron mejoras estadísticamente significativas en los problemas psicopatológicos y las actividades de la vida diaria medidos con las escalas NPI e índice de Katz respectivamente.

Hassandra et al. (28) realizaron una intervención con condición A (ciclismo en entorno de laboratorio mientras realizan cálculos matemáticos orales de un solo digito) y condición B (tarea de ciclismo en entorno virtual mientras realizan cálculos que aparecen dentro de la aplicación de RV) con 27 personas en 1 sesión de 60 minutos. Aplicaron un cuestionario propio y analizaron las variables aceptabilidad, usabilidad y tolerabilidad. Los resultados indican preferencia por la condición $B(P<0.001)$, una puntuación de usabilidad de 77.6 (umbral $75 / 100$ ) y no se hallaron efectos adversos, lo que indica tolerabilidad satisfactoria.

Chen et al. (29) realizaron una intervención en 3 grupos (Normal $(n=16), D C L(n=10)$, Demencia moderada-grave $(n=11))$ para jugar a los juegos Whack-a-mole y Hit-the-ball. Participaron 37 personas en 1 sesión (tiempo indeterminado). Aplicaron la escala MMSE y analizaron las variables precisión y tiempo de reacción. Los resultados muestran que los grupos DCL y demencia moderada-grave tienen tiempos de reacción mayores y menor tasa de precisión que el grupo normal.

Iliadou et al. (30) realizaron una intervención en 2 grupos (Normal $(n=43), D C L(n=33))$ con 3 tareas VST "Virtual Supermarket" sobre una muestra de 76 personas en 1 sesión de 30 minutos. Aplicaron las escalas EEG y MoCA, y analizaron el tiempo en completar prueba. Los resultados indican que el grupo DCL tarda mayor tiempo en completar las 3 tareas que el grupo de adultos sanos $(p<0.05)$.

Skikos et al. (31) realizaron una intervención de 10 tareas con 10 juegos diferentes sobre 6 personas en 10 sesiones de 30 minutos. Aplicaron las escalas MoCA, MMSE, Escala de usabilidad del sistema (SUS) y Escala Likert de 5 ítems, y analizaron el tiempo de finalización de la prueba. Los resultados son 89.4 sobre 100 en la escala SUS y una mejora en el tiempo medio de finalización de todos los usuarios.

Ge et al. (32) realizaron una intervención de 2 tareas VST alternando 40s de juego y 20s de descanso (7 veces) sobre 37 personas en 1 sesión con 2 tareas de 440 segundos. Aplicaron las escalas MMSE y sistema de infrarrojo cercano multicanal, y analizaron la activación cerebral con diferentes tipos de ondas. Los resultados indican que el entrenamiento cognitivo puede ralentizar el deterioro cognitivo. 
Bojan et al. (33) realizaron una intervención para jugar a 15 juegos de COSMA con 30 personas en 1 sesión de 2 horas y media. Aplicaron la escala Positive and Negative Affect Schedule (PANAS), y analizaron las variables interés, nivel de alerta, excitación, nerviosismo e irritabilidad. Los resultados muestran que los efectos positivos aumentaron en los 2 grupos después de la intervención ( $p=0.041$ ) y los efectos negativos disminuyeron en los 2 grupos después de la intervención ( $p=0.001$ ).

En la tabla 2 se resumen los principales resultados tras el análisis de los artículos.

Tabla 2. Principales resultados tras el análisis de los artículos

\begin{tabular}{|c|c|c|c|}
\hline Autor & Año & $\begin{array}{l}\text { Edad } \\
\text { (años) }\end{array}$ & Resultados \\
\hline $\begin{array}{l}\text { 1. Tziraki et al. } \\
\text { 5. Yun et al. } \\
\text { 6. Burdea et al. } \\
\text { 12. Skikos et al. }\end{array}$ & $\begin{array}{l}2017 \\
2020 \\
2015 \\
2021\end{array}$ & $\begin{array}{c}>65 \\
\geq 65 \\
55-73 \\
72 \text { (media) }\end{array}$ & $\begin{array}{l}\text { Mejoras en los tiempos } \\
\text { de finalización y } \\
\text { reacción, así como en la } \\
\text { velocidad de } \\
\text { procesamiento }\end{array}$ \\
\hline $\begin{array}{l}\text { 2. Fasilis et al. } \\
\text { 8. Swinnen et al. }\end{array}$ & $\begin{array}{l}2018 \\
2021\end{array}$ & $\begin{array}{c}73.6 \\
(\text { media) } \\
>65\end{array}$ & $\begin{array}{l}\text { Mejora de las funciones } \\
\text { ejecutivas, así como en la } \\
\text { atención y memoria de } \\
\text { trabajo visoespacial }\end{array}$ \\
\hline $\begin{array}{l}\text { 6. Burdea et al. } \\
\text { 8. Swinnen et al. }\end{array}$ & $\begin{array}{l}2015 \\
2021\end{array}$ & $\begin{array}{l}55-73 \text { años } \\
>65 \text { años }\end{array}$ & $\begin{array}{c}\text { Mejora en los síntomas } \\
\text { de depresión }\end{array}$ \\
\hline $\begin{array}{l}\text { 4. Amaefule et al. } \\
\text { 9. Hassandra et al. } \\
\text { 12. Skikos et al. }\end{array}$ & $\begin{array}{l}2020 \\
2021 \\
2021\end{array}$ & $\begin{array}{c}60-85 \\
73.22 \\
\text { (media) } \\
72 \text { (media) }\end{array}$ & $\begin{array}{l}\text { Grado considerable de } \\
\text { inmersión en la tarea, así } \\
\text { como en la usabilidad }\end{array}$ \\
\hline $\begin{array}{l}\text { 3. Robert et al. } \\
\text { 10. Chen et al. } \\
\text { 11. Iliadou et al. }\end{array}$ & $\begin{array}{l}2020 \\
2021 \\
2021\end{array}$ & $\begin{array}{c}79.4 \\
\text { (media) } \\
75.38 \\
\text { (media) } \\
66.09 \\
\text { (media) }\end{array}$ & $\begin{array}{c}\text { Posibles mejoras en } \\
\text { rendimiento cognitivo, } \\
\text { tiempo de reacción y } \\
\text { tiempo en completar la } \\
\text { tarea }\end{array}$ \\
\hline
\end{tabular}

\section{DISCUSIÓN}

En base a los artículos analizados en esta revisión sistemática comprobamos que los "serious games" comienzan a consolidarse como una alternativa a tener en cuenta a los tratamientos tradicionales de estimulación cognitiva en el cribado y tratamiento de la demencia, pero existen una serie de factores que dificultan la obtención de conclusiones de peso y rigor científico. A continuación, pasamos a describir y analizar dichos factores.

Uno de los principales obstáculos que nos encontramos a la hora de unificar resultados es la falta de homogeneidad que existe entre los artículos, puesto que las intervenciones realizadas difieren mucho entre los estudios. La duración y frecuencia de las intervenciones realizadas es muy diversa. Encontramos intervenciones cuya duración fue una única sesión de 30 minutos (24) (30) y otras cuya duración fue de 10-12 semanas (22) (20). La frecuencia de las intervenciones analizadas osciló entre la sesión única de 30 minutos anteriormente mencionada, a 4 veces por semana. Sin embargo, tras completar el análisis, no hayamos pruebas estadísticamente significativas de que las intervenciones con mayor duración y frecuencia arrojaran mejores resultados en los parámetros analizados. Otro de los obstáculos hallado hace referencia a los tamaños de muestra con los que cuentan los estudios, los cuales oscilaron entre 6 sujetos (31) y 76 sujetos (30), siendo la media de 28 sujetos, lo cual, si tenemos en cuenta la división entre grupo control y grupo tratamiento, consideramos una muestra pequeña para lograr potencial estadístico y resultados extrapolables a la población general. 


\section{APLICACIÓN DE "SERIOUS GAMES” EN EL TRATAMIENTO DE LA DEMENCIA: REVISIÓN SISTEMÁTICA}

Por otro lado, encontramos la diversidad de "serious games" utilizados en los artículos incluidos en esta revisión y la diversidad de variables que analizan. Algunos de ellos se encuentran en fase de viabilidad formando parte de estudios piloto (20) (24) (33). Algunas de las variables que analizan son las siguientes: velocidad de procesamiento, tiempo de reacción, velocidad motora, nivel de alerta y excitación. Las herramientas estandarizadas utilizadas en ellos también son diversas, entre las más usadas encontramos MMSE y MoCA. Debido a la diversidad de "serious games" utilizados y a la diversidad de variables utilizadas, se hace difícil Ilegar a conclusiones generales acerca de sus beneficios en sujetos con demencia. Se hace necesario la creación de algún tipo de herramienta estandarizada especializada en el campo de la aplicación de "serious games" en sujetos con demencia. De este modo, los resultados serían más homogéneos y fáciles de analizar y comparar.

Otro punto a destacar tras el análisis de los artículos es la diversidad de profesionales de la salud que encontramos en los estudios y sus correspondientes intervenciones. Se ha echado en falta la figura del Terapeuta Ocupacional, la cual solo aparece en uno de los estudios (24), siendo éste un estudio de viabilidad. Su labor, además de la de "tester", fue la de supervisar al resto de usuarios durante el desarrollo de la prueba ofreciendo asistencia verbal y física a los usuarios que mostraban dificultades en la comprensión de instrucciones o la ejecución de la tarea. La figura del Terapeuta Ocupacional podría aportar un salto cualitativo en lo que a este campo refiere, puesto que una de las áreas de trabajo recogida en el marco de la "American Occupational Therapy Association" (AOTA) (2) es el juego, aplicado como herramienta terapéutica. El Terapeuta Ocupacional podría participar en todas las fases del proceso de creación de herramientas terapéuticas basadas en "serious games", desde el diseño, en el que trabajaría en conjunto con otros profesionales, como ingenieros informáticos o desarrolladores de aplicaciones, para asegurar un contenido de calidad que responda a las necesidades y particularidades tanto de la patología a tratar como las de los propios sujetos futuros usuarios del "serious game", pasando por las fases de desarrollo, en las que aportaría su experiencia de campo en el trabajo con usuarios, hasta las fases de aplicación y posterior evaluación, en las que se aseguraría que la interacción entre usuario y "serious game", sea la adecuada acorde a las características particulares de cada uno.

Como aspectos relevantes a la hora de que un Terapeuta Ocupacional opte por usar "serious games" en el tratamiento de la demencia, podríamos destacar: a) el grado considerable de inmersión en la tarea (28), lo cual supone una gran ventaja respecto a la estimulación cognitiva con material convencional, en la cual el profesional ha de ocuparse de mantener la motivación en la tarea; b) la mejora de la atención y memoria de trabajo (27), requisitos indispensables para el correcto desarrollo de una sesión de estimulación cognitiva; c) la mejora del tiempo medio de finalización de la tarea (31), la cual facilitaría sesiones individuales con mayor variedad de intervenciones y materiales; y d) que el entrenamiento cognitivo puede ralentizar el deterioro cognitivo (32), lo cual nos puede ayudar a frenar determinados signos y síntomas que dificultan la realización satisfactoria de una sesión individual. Por otro lado, al tratarse la mayoría de juegos y/o dispositivos que los usuarios podrían utilizar en su entorno habitual, podrían reforzarse y/0 aumentarse los beneficios que aportan las sesiones de Terapia Ocupacional presenciales. También, podría servir como sistemas de monitorización que arrojen información acerca del usuario más allá de las consultas programadas.

Algunos de los artículos analizados utilizaron medidas de usabilidad (26) (28) (31) para comprobar el manejo y desempeño de los usuarios en la utilización del "serious game". En el caso de Vallejo, et al. (26) no se hallaron diferencias significativas de usabilidad entre el grupo control y tratamiento, lo que implica que las tareas a realizar fueron claras y concisas, y no necesitaron explicaciones adicionales para su realización. En el caso de Hassandra, et al. (28) la puntuación de usabilidad fue alta, lo que implica que un buen diseño tanto del "serious game" como de su contenido garantiza una experiencia positiva por parte de los usuarios. En el caso de Skikos et al. (31) también se obtuvo una puntuación alta de usabilidad. Por otro lado, en el caso de Robert et al. (22), se produjo un aumento de la apatía en el grupo no activo. La participación de la figura del Terapeuta Ocupacional en la fase piloto de desarrollo podría aportar una mejora en la experiencia final del usuario puesto que en su práctica 
profesional cuenta con la graduación de la actividad, que consta de la descomposición de la actividad en pequeñas tareas, para analizar los pormenores y dificultades a las que el usuario se puede enfrentar.

Existen efectos positivos diversos en el uso de "serious games" en el tratamiento de la demencia, como es el caso de Fasilis et al. (21), en el que existe mejora de la memoria y de las funciones ejecutivas, o el caso de Burdea et al. (25), en el que encontramos mejoría en la toma de decisiones, así como disminución en los niveles de depresión. También en el caso de Swinnen et al. (27), en el que encontramos múltiples y diversas mejoras como: mejora en el funcionamiento de los miembros inferiores, mejora en la atención y en la memoria de trabajo visoespacial y, mejora en los síntomas de depresión, pero no se hallaron mejoras en las actividades de la vida diaria. Por lo tanto, dichos efectos positivos, se podrían traducir en mejora de la calidad de vida e independencia del usuario si, además, la temática de los juegos estuviera enfocada a la autonomía personal, la cual, además de enriquecer la gama de juegos, sería una herramienta valiosa a la hora de intervenir en las áreas ocupacionales por parte de los Terapeutas Ocupacionales.

Existen otras revisiones sistemáticas acerca del uso de "serious games" en el tratamiento de la demencia, como es el caso de Huansheng Ning et al. (34), la cual aboga por presentar una arquitectura de diseño y categorización más clara y concisa que la actual, así como un modelo de evaluación que garantice que el juego que se va a utilizar cumple con las características y necesidades que presenta el usuario potencial. Por otro lado, en el caso de Sardi et al. (19), remarca que uno de los ámbitos más estudiados en la utilización de "serious games" en el campo de la salud es la actividad física, por ello se hace necesaria una mayor investigación en campos como la estimulación cognitiva o el tratamiento de enfermedades crónicas. También hace hincapié en que la mecánica de juego es repetitiva haciendo uso de recompensas y retroalimentación. Dichas recompensas y retroalimentación han de usarse de modo combinado con otro tipo de elementos motivadores para evitar que el usuario pierda motivación e interés por la tarea.

Como último punto de la discusión podemos destacar que las generaciones nacidas hace un par de décadas y las venideras son generaciones nativas digitales, por lo tanto, el uso de tecnologías en el campo del cuidado y la salud cobrará una especial importancia de aquí en adelante, pudiendo aprovechar todo el potencial que nos ofrecen las nuevas tecnologías en campos como la informática y la telefonía móvil, para desarrollar y crear experiencias de prevención y cuidado de la enfermedad cada vez más personalizadas y accesibles a la mayoría de la población, aprovechando una de sus ventajas notables como es garantizar el compromiso regular de los usuarios y aumentar su grado de inmersión y compromiso terapéutico. Además, el uso de "serious games" aumenta la motivación de los usuarios y disminuye síntomas apáticos y de depresión (27) (25), lo que beneficia al proceso terapéutico de tratamiento de enfermedades crónicas como es la demencia en este caso.

\section{CONCLUSIONES}

Tras el estudio de los artículos seleccionados en esta revisión consideramos que se hace necesaria una mayor homogeneidad tanto en la duración como en la frecuencia de las intervenciones que comprendan el uso de "serious games" aplicados a la demencia. Se necesitan estudios con mayor tamaño de muestra para obtener resultados con mayor potencia estadística. Además una mayor homogeneidad en las variables utilizadas en los estudios que comprenden el uso de "serious games" aplicados en la demencia permitiría facilitar la comparación de resultados.

Ha quedado patente que la figura del Terapeuta Ocupacional, respecto al uso de "serious games" en la literatura analizada, no tiene la relevancia que a nuestro juicio debería, y que consideramos que desde nuestro colectivo debe trabajarse esta herramienta con un interesante potencial terapéutico en las distintas áreas ocupacionales, más allá del área del juego.

A nuestro juicio la utilización de "serious games" aplicados en la demencia creará experiencias de prevención y cuidado de la enfermedad más personalizadas y accesibles a la mayoría de la población. 


\section{APLICACIÓN DE "SERIOUS GAMES” EN EL TRATAMIENTO DE LA DEMENCIA: REVISIÓN SISTEMÁTICA}

\section{REFERENCIAS BIBLIOGRÁFICAS}

(1) Demencia. https://www.who.int/es/news-room/fact-sheets/detail/dementia. Accedido: 25/11/2021.

(2) Marco de Trabajo de Terapia Ocupacional. http://bases.cortesaragon.es/bases/NDocumen.nst/0/2930ff892352fe31c12573a2002e931c/\$FILE/Marco_d e_Trabajo_de_Terapia_Ocupacional.pdf. Accedido: 25/11/2021

(3) Consenso español sobre demencias: http://www.sepsiq.org/file/Publicaciones/Consenso\%20espa\%C3\%B101\%20sobre\%20demencias.pdf. Accedido: 25/11/2021.

(4) Ory MG, Hoffman RR, Yee JL, Tennstedt S, Schulz R. Prevalence and impact of caregiving: a detailed comparison between dementia and nondementia caregivers. Gerontologist. 1999; 39(2):177-85.

(5) Grant I, Adler KA, Patterson TL, Dimsdale JE, Ziegler MG, Irwin MR. Health consequences of Alzheimer's caregiving transitions: effects of placement and bereavement. Psychosom Med. 2002; 64(3):477-86.

(6) Formiga F, Robles MJ, Fort I. Demencia, una enfermedad evolutiva: demencia severa. Identificación de demencia terminal. Rev Esp Geriatr Gerontol. 2009; 44:2-8.

(7) Viadero CF. Demencia severa, avanzada y cuidados paliativos. 2012; 97.

(8) López Locanto Ó. Tratamiento farmacológico de la enfermedad de Alzheimer y otras demencias. Archivos de Medicina Interna. 2015; 37(2):61-7.

(9) Michael DR, Chen SL. Serious Games: Games That Educate, Train, and Inform. Muska \& Lipman/PremierTrade; 2005.

(10) Stapleton AJ. Serious games: Serious opportunities. En: In Australian Game Developers Conference, Academic. 2004.

(11) Tárraga L, Boada M, Modinos G, Espinosa A, Diego S, Morera A, et al. A randomised pilot study to assess the efficacy of an interactive, multimedia tool of cognitive stimulation in Alzheimer's disease. J Neurol Neurosurg Psychiatry. 2006; 77(10):1116-21.

(12) Ricciardi F, Paolis LTD. A Comprehensive Review of Serious Games in Health Professions. International Journal of Computer Games Technology. 2014.

(13) Djaouti D, Alvarez J, Jessel J-P, Rampnoux 0. Origins of Serious Games. En Serious Games and Edutainment Applications. London: Springer; 2011.

(14) Lehmann ED. Interactive educational simulators in diabetes care. Medical Informatics. 1997; 22(1):47-76.

(15) Brown SJ, Lieberman DA, Germeny BA, Fan YC, Wilson DM, Pasta DJ. Educational video game for juvenile diabetes: results of a controlled trial. Med Inform (Lond). 1997; 22(1):77-89.

(16) Gajardo J J, Aravena C JM. ¿Cómo aporta la terapia ocupacional en el tratamiento de las demencias? Revista chilena de neuro-psiquiatría. 2016; 54(3):239-49.

(17) Rodriguez J, Gajardo J. Sobre la contribución de la terapia ocupacional en el manejo no farmacológico de Ios síntomas psicológicos y conductuales asociados a la demencia. Revista Chilena de Terapia Ocupacional. 2012; 12(2).

(18) Moyà Alcover G, Perales F, Varona J. Rehabilitación motivacional basada en la utilización de serious games Motivational Rehabilitation using Serious Games. Virtual Archaeology Review. 2013; 4:167.

(19) Sardi L, Idri A, Fernández-Alemán JL. A systematic review of gamification in e-Health. Journal of Biomedical Informatics. 2017; 71:31-48.

(20) Tziraki C, Berenbaum R, Gross D, Abikhzer J, Ben-David BM. Designing Serious Computer Games for People With Moderate and Advanced Dementia: Interdisciplinary Theory-Driven Pilot Study. JMIR Serious Games. 2017; 5(3).

(21) Fasilis Th, Patrikelis P, Siatouni A, et al. A pilot study and brief overview of rehabilitation via virtual environment in patients suffering from dementia. Psychiatriki. 2018; 29(1):42-51. 
(22) Robert P, Manera V, Derreumaux A, Ferrandez Y Montesino M, Leone E, Fabre R, et al. Efficacy of a Web App for Cognitive Training (MeMo) Regarding Cognitive and Behavioral Performance in People With Neurocognitive Disorders: Randomized Controlled Trial. J Med Internet Res. 2020; 22(3).

(23) Amaefule C0, Lüdtke S, Kirste T, Teipel SJ. Effect of Spatial Disorientation in a Virtual Environment on Gait and Vital Features in Patients with Dementia: Pilot Single-Blind Randomized Control Trial. JMIR Serious Games. 2020; 8(4).

(24) Yun SJ, Kang M-G, Yang D, Choi Y, Kim H, Oh B-M, et al. Cognitive Training Using Fully Immersive, Enriched Environment Virtual Reality for Patients With Mild Cognitive Impairment and Mild Dementia: Feasibility and Usability Study. JMIR Serious Games. 2020; 8(4).

(25) Burdea G, Polistico K, Krishnamoorthy A, House G, Rethage D, Hundal J, et al. Feasibility study of the BrightBrainer ${ }^{T M}$ integrative cognitive rehabilitation system for elderly with dementia. Disabil Rehabil Assist Technol. 2015; 10(5):421-32.

(26) Vallejo V, Wyss P, Rampa L, Mitache AV, Müri RM, Mosimann UP, et al. Evaluation of a novel Serious Game based assessment tool for patients with Alzheimer's disease. PLoS One. 2017; 12(5).

(27) Swinnen N, Vandenbulcke M, de Bruin ED, Akkerman R, Stubbs B, Firth J, et al. The efficacy of exergaming in people with major neurocognitive disorder residing in long-term care facilities: a pilot randomized controlled trial. Alzheimers Res Ther. 2021; 13.

(28) Hassandra M, Galanis E, Hatzigeorgiadis A, Goudas M, Mouzakidis C, Karathanasi EM, et al. Virtual Reality App for Physical and Cognitive Training of Older People With Mild Cognitive Impairment: Mixed Methods Feasibility Study. JMIR Serious Games. 2021; 9(1):e24170.

(29) Chen Y-T, Hou C-J, Derek N, Huang S-B, Huang M-W, Wang Y-Y. Evaluation of the Reaction Time and Accuracy Rate in Normal Subjects, MCl, and Dementia Using Serious Games. Applied Sciences. 2021; 11(2):628.

(30) Iliadou P, Paliokas I, Zygouris S, Lazarou E, Votis K, Tzovaras D, et al. A Comparison of Traditional and Serious Game-Based Digital Markers of Cognition in Older Adults with Mild Cognitive Impairment and Healthy Controls. Journal of Alzheimer's Disease. 2021; 79(4):1747-59.

(31) Skikos G, Goumopoulos C. A Tablet-Based Game Tool for Cognition Training of Seniors with Mild Cognitive Impairment. En International Conference on Database Systems for Advanced Applications. 2021; 355-364. Springer, Cham.

(32) Ge R, Wang Z, Yuan X, Li Q, Gao Y, Liu H, et al. The Effects of Two Game Interaction Modes on Cortical Activation in Subjects of Different Ages: A Functional Near-Infrared Spectroscopy Study. IEEE Access. 2021; 9:11405-15.

(33) Bojan K, Stavropoulos TG, Lazarou I, Nikolopoulos S, Kompatsiaris I, Tsolaki M, et al. The effects of playing the COSMA cognitive games in dementia. IJSG. 2021; 8(1):45-58.

(34) Ning H, Li R, Ye X, Zhang Y, Liu L. A Review on Serious Games for Dementia Care in Ageing Societies. IEEE J Transl Eng Health Med. 2020;8. 
